\title{
Inhibition of the Tyrosine Phosphatase SHP-2 Suppresses Angiogenesis in vitro and in vivo
}

\author{
Hanna Mannell ${ }^{a} \quad$ Nicole Hellwig ${ }^{a} \quad$ Torsten Gloe $^{a} \quad$ Christian Plank $^{c}$ \\ Hae-Young Sohn $^{b}$ Leopold Groesser ${ }^{\mathrm{a}} \quad$ Barbara Walzog $^{\mathrm{a}} \quad$ Ulrich Pohl $^{\mathrm{a}}$ \\ Florian Krötz ${ }^{a, b}$ \\ Institute of a Physiology and ${ }^{\mathrm{b}}$ Cardiology, Medical Policlinic, Ludwig Maximilians University, and \\ 'Department of Experimental Oncology and Therapeutic Research, Technical University, Munich, Germany
}

\section{Key Words}

Angiogenesis • Endothelial cells • FGF-2 • PI3-K • SHP-2 • Tyrosine phosphatase

\begin{abstract}
Endothelial cell survival is indispensable to maintain endothelial integrity and initiate new vessel formation. We investigated the role of SHP-2 in endothelial cell survival and angiogenesis in vitro as well as in vivo. SHP-2 function in cultured human umbilical vein and human dermal microvascular endothelial cells was inhibited by either silencing the protein expression with antisense-oligodesoxynucleotides or treatment with a pharmacological inhibitor (PtpI IV). SHP2 inhibition impaired capillary-like structure formation $(p<$ $0.01 ; n=8)$ in vitro as well as new vessel growth ex vivo $(p<$ $0.05 ; n=10$ ) and in vivo in the chicken chorioallantoic membrane $(p<0.01, n=4)$. Additionally, SHP-2 knock-down abrogated fibroblast growth factor 2 (FGF-2)-dependent endothelial proliferation measured by MTT reduction $(p<0.01$; $n=12$ ). The inhibitory effect of SHP-2 knock-down on vessel growth was mediated by increased endothelial apoptosis (annexin $\mathrm{V}$ staining, $\mathrm{p}<0.05, \mathrm{n}=9$ ), which was associated with reduced FGF-2-induced phosphorylation of phosphatidylinositol 3-kinase (PI3-K), Akt and extracellular regulated kinase 1/2 (ERK1/2) and involved diminished ERK1/2 phosphorylation after PI3-K inhibition $(n=3)$. These results sug-
\end{abstract}

gest that SHP-2 regulates endothelial cell survival through PI3-K-Akt and mitogen-activated protein kinase pathways thereby strongly affecting new vessel formation. Thus, SHP2 exhibits a pivotal role in angiogenesis and may represent an interesting target for therapeutic approaches controlling vessel growth.

Copyright $\odot 2007$ S. Karger AG, Basel

\section{Introduction}

The Src homology 2 domain containing tyrosine phosphatase 2, SHP-2 (also called SH-PTP2, SH-PTP3, PT$\mathrm{P} 2 \mathrm{C}, \mathrm{PTP} 1 \mathrm{D}$ or Syp), is ubiquitously expressed and possibly also functions as an adaptor protein [1-4]. During the development of hematopoietic cells, it maintains the critical balance between apoptosis, proliferation and differentiation [5-7]. In endothelial cells, SHP-2 interacts with adhesion molecules, such as PECAM-1 and VE-cadherin, thereby participating in mechanotransduction and cell motility [8-10]. Mobilization of endothelial cells is a prerequisite for angiogenesis, during which the prevention of endothelial apoptosis or anoikis (programmed cell death due to detachment from the extracellular matrix) [11-13] is of major importance in order to enable the formation of new vessels from already existing ones.

\begin{tabular}{ll}
\hline KARGER & ( 2007 S. Karger AG, Basel \\
1018-1172/08/0452-0153\$24.50/0 \\
$\begin{array}{l}\text { Fax +41 61 306 1234 } \\
\begin{array}{l}\text { E-Mail karger@karger.ch } \\
\text { www.karger.com }\end{array}\end{array}$ & $\begin{array}{l}\text { Accessible online at: } \\
\text { www.karger.com/jvr }\end{array}$
\end{tabular}

Dr. Florian Krötz

Cardiology, Medical Policlinic, Ludwig Maximilians University

Ziemssenstrasse 1, DE-80336 Munich (Germany)

Tel. +498921807 5384, Fax +4989218075378

E-Mail Florian.Kroetz@med.uni-muenchen.de 
The outstanding role of fibroblast growth factor 2 (FGF-2) in angiogenesis $[11,14,15]$ is reflected by its high local concentrations during wound healing $[16,17]$. FGF2 has also been suggested to facilitate the angiogenic growth response of endothelial cells during ischemia [18]. Furthermore, apart from promoting endothelial proliferation and migration, FGF-2 is one of the strongest mediators of endothelial cell survival $[11,15]$ and induces tube-like structure formation $[14,17,18]$. A potential role of SHP-2 in FGF-2-dependent endothelial cell signaling, influencing the FGF-2-dependent angiogenic response, has not yet been explored. In addition, it is unclear whether SHP-2 is involved in endothelial cell survival and new vessel formation and thus could be important for angiogenesis in vivo.

Therefore, we investigated whether SHP-2 is involved in human endothelial cell survival, tube-like structure formation in vitro, vessel sprouting ex vivo and whether a functional SHP-2 is necessary for the formation of new vessels in vivo. Finally, we explored if SHP-2 influences the FGF-2-dependent activation of the anti-apoptotic signaling pathways phosphatidylinositol 3-kinase (PI3-K)/ Akt and mitogen-activated protein kinase (MAPK) in endothelial cells.

\section{Materials and Methods}

\section{Chemicals}

Monoclonal SHP-2 antibody, polyclonal phospho-Akt 1/2/3 antibody and actin antibody were from Santa Cruz Biotechnology (Heidelberg, Germany). Monoclonal phospho-42/44 antibody was from Cell Signaling (Frankfurt am Main, Germany). Monoclonal phosphotyrosine antibody (clone 4G10) was from Upstate (Hamburg, Germany) and monoclonal GAPDH antibody was from Chemicon (Chandlers Ford, UK). Horseradish peroxidaseconjugated antibodies and the pharmacological SHP-2 inhibitor (PtpI IV) were from Calbiochem (Darmstadt, Germany). FGF-2 was purchased from Tebu-bio (Offenbach, Germany). All other chemicals were from Sigma-Aldrich (Taufkirchen, Germany).

\section{Cell Lines and Cell Culture}

Human umbilical vein endothelial cells (HUVEC) were isolated and cultured as described previously [19]. Human dermal microvascular endothelial cells (HMEC) were provided by Ades et al. [20] and cultured in M199 media supplemented with $10 \%$ FCS, $10 \%$ endothelial growth media (PromoCell, Heidelberg, Germany) and $1 \%$ penicillin/streptomycin.

\section{Antisense-Oligodesoxynucleotide Transfer}

Endothelial SHP-2 protein was knocked down by phosphorothioate-modified oligodesoxynucleotide (ODN) transfection using the previously described magnetofection technique [21-23]. Control transfections were performed using an ODN with a random sequence not aligning to any human mRNA (Rdm-ODN).
Oligonucleotide sequences were as follows: SHP-2 antisense (AS)-ODN: 5'-ctccgcgatgtcatgttcct-3', Rdm-ODN: 5'-cccttatttactactttcgc-3' (both from MWG Biotech, Ebersberg, Germany). To control for efficient transfection, Cy-3 fluorescence-labeled ODN were transfected and sufficient efficiency was controlled for by flow-cytometric analysis (>80\%) as described [21].

\section{Cell Extracts for Immunoblotting and Western Blot Analysis}

Protein lysates were prepared and protein content quantified according to a previous method [24]. Lysates were subjected to Western blot analysis as shown [24].

\section{MTT Assay}

Cell proliferation and cell survival of HMEC was measured by the reduction of methylthiazoletetrazolium, MTT $(0.5 \mathrm{mg} / \mathrm{ml})$, as described by Mosmann et al. [25] with minor modifications, as well as by measuring protein concentration with the BCA kit (Pierce, Bonn, Germany) according to the supplier's protocol.

\section{Cell Viability and Apoptosis Measurements}

Cell cycle states of HUVEC were assessed by flow-cytometric measurement of propidium iodide-stained DNA according to Crompton et al. [26], with minor modifications, to analyze cell viability. To assess the amount of intact, yet apoptotic cells, an annexin V/propidium iodide assay was performed whereby double staining against annexin $\mathrm{V}$ together with propidium iodide separates cells currently undergoing apoptosis, but which are not necrotic, from cells that have died of necrosis. The assay was performed according to the manufacturer's instructions (Annexin V Apoptosis detection kit 1, BD Biosciences, Heidelberg, Germany) and annexin $\mathrm{V}$ positive but propidium iodide negative cells were detected by flow cytometry. Cell viability was assessed by trypan blue exclusion. Cells were detached non-enzymatically from the cell dish, diluted $1: 1$ in PBS and $0.2 \%$ trypan blue, incubated for 2 min and subsequently counted with fast-read counting chambers (Biosigma, Cona, Italy). Cells stained blue were counted as non-viable.

\section{Immunoprecipitation of $p 85$}

Immunoprecipitations were performed using $\mu$ MACS Protein G MicroBeads and MACS separation columns from Miltenyi Biotec (Bergisch Gladbach, Germany) according to the manufacturer's protocol. The activity of PI3-K was assessed by immunoblotting against phosphotyrosine.

\section{Activity Assays}

For extracellular regulated kinase 1/2 (ERK1/2) and Akt activity, cells were treated with $10 \mathrm{ng} / \mathrm{ml} \mathrm{FGF}-2$ for $10 \mathrm{~min}$, lysed and the lysate submitted to Western blotting as described above. Membranes were incubated with the respective phospho-antibodies. An anti-GAPDH antibody was used to ensure equal protein loading.

\section{Capillary-Like Structure Assay}

Confluent HUVEC were treated with PtpI IV $(2 \mu \mathrm{M})$ [27] or DMSO (sham group) for $6 \mathrm{~h}$, SHP-2 AS-ODN or Rdm-ODN $24 \mathrm{~h}$ prior to trypsination. The cells were then seeded onto a 24 -well plate filled with growth factor-reduced Matrigel (BD Biosciences, Erembodegem, Belgium) and left to form capillary-like structures overnight in growth factor-rich medium. Pictures were taken with an LSM 410 microscope, and the capillary-like structures in each sector were counted. 
Aortic Ring Sprouting Assay

Adult Black 6 mice were euthanized with an overdose of sodium pentobarbital (Narcoren ${ }^{\mathrm{TM}}$; Merial, Hallbergmoos, Germany) and aortae were dissected. After rinsing with Tutofusin Voll-E ${ }^{\circledR}$ (Baxter, Unterschleissheim, Germany), aortae were cut into rings and embedded in growth factor-reduced Matrigel. They were then treated with and kept in either $2 \mu \mathrm{M}$ PtpI IV or DMSO (sham group) in growth media. The aortic rings were left for $96 \mathrm{~h}$. Pictures were taken with an LSM 410 microscope. To measure angiogenesis, the bifurcations of the new vessels were counted manually.

\section{Chick Chorioallantoic Membrane Assay}

The effect of SHP-2 inhibition on angiogenesis in vivo was investigated on the chorioallantoic membrane (CAM) of 10-day-old chicken embryos, as previously described by Brooks et al. [28], with minor modifications. Chicken embryos were candled to visualize the existing blood vessels of the CAM and a spot where no vessels were growing was chosen. At this spot, the CAM was next separated from the shell generating a false air sac above which a small window was cut out. A filter disc soaked in FGF-2/VEGF-A (both $50 \mathrm{ng} / \mathrm{ml}$ ) and DMSO (sham solution), in FGF-2/VEGF-A $(50 \mathrm{ng} / \mathrm{ml})$ and PtpI IV $(2 \mu \mathrm{M})$ or in DMSO (sham solution) was carefully placed on top of the CAM. The window was sealed with adhesive tape and the embryos were left in a humidified incubator for 3 days. The sham solution and PtpI IV were applied every day to the filter discs. Before removal of the filter disc and surrounding CAM tissue, the CAM was fixed in formalin (3.7\%). The removed filter and CAM tissue were washed in PBS before photos of the CAM were taken with an AxioCam microscope (Zeiss, Jena, Germany). As an index of angiogenesis, the number of branching points (bifurcations) of the vessels formed per visual field was counted.

\section{Statistical Analysis}

Data were analysed using Student's t test, one-way ANOVA or a rank sum test, as appropriate. All data are presented as means \pm SEM. Results were considered significant at an error probability level of $\mathrm{p}<0.05$.

\section{Results}

\section{SHP-2 Protein Knock-Down Decreases Endothelial Cell MTT Reduction}

Previous studies using AS-ODN combined with magnetofection in HUVEC have shown a transfection efficiency of $>80 \%$ compared to conventional transfection [21]. Compared to Rdm-ODN treatment, SHP-2 AS-ODN resulted in a marked decrease in SHP-2 protein content in endothelial cells (HUVEC and HMEC) already after $6 \mathrm{~h}$, and was still seen $24 \mathrm{~h}$ following magnetofection (fig. $1 \mathrm{a}$, all $n=3$ ). Therefore, the following experiments were performed during or 6-24 $\mathrm{h}$ following transfection. A downregulation of SHP-2 protein expression precluded the FGF-2-enhanced MTT reduction, a substrate used to as- sess the amount of viable cells and cellular proliferation. Whereas FGF-2 stimulation of Rdm-ODN-treated cells resulted in a significant increase in MTT reduction compared to non-stimulated cells ( $\mathrm{p}<0.05 ; \mathrm{n}=9$, HMEC, fig. 1b) and thus proliferation of endothelial cells, SHP-2 AS-ODN treatment abolished this effect by $41 \pm 15 \%(24 \mathrm{~h}$ after stimulation, $\mathrm{p}<0.01 ; \mathrm{n}=12$, HMEC, fig. $1 \mathrm{~b}$ ) thereby reaching values below the starting point $(t=0 \mathrm{~h})$, indicating a reduction in cell number. To verify these data, HMEC protein concentration was measured after ODN treatment confirming the previous results, as SHP-2 knock-down followed by FGF-2 stimulation reduced the protein content by $62 \%$ ( $\pm 10 \% 48 \mathrm{~h}$ after stimulation with FGF-2, $\mathrm{p}<0.01$; $n=9$, fig. 1c) compared to Rdm-ODN treatment $(n=6)$.

\section{Downregulation of SHP-2 Protein Expression Affects} Endothelial Cell Viability and Induces Apoptosis

To investigate if the decreased MTT reduction following SHP-2 knock-down was due to apoptosis, HUVEC DNA was stained with propidium iodide, and the amount of cells with lower amounts of DNA than a cell situated in the G0 stage of the cell cycle (SubG0) was detected. As these cells contain less DNA than a resting cell, due to apoptosis-induced DNA fragmentation, this fraction was measured as an index of apoptosis. Six hours after transfection, SHP-2 AS-ODN treatment slightly increased the amount of cells in SubG0 compared to Rdm-ODN-transfected cells. Twenty-four hours following transfection, this difference reached significance $(25 \pm 5 \%$ of all cells in SubG0 with SHP-2 AS-ODN in comparison to $10 \pm$ $0.8 \%$ with Rdm-ODN, fig. $2, \mathrm{p}<0.01 ; \mathrm{n}=6$, HUVEC). To confirm SHP-2 involvement in endothelial apoptosis, cells were stained against the proapoptotic marker annexin V. Following SHP-2 knock-down, there was a significant increase in annexin $\mathrm{V}$-positive cells compared to Rdm-ODN $(23 \pm 7 \% 6 \mathrm{~h}$ after transfection, $\mathrm{p}<0.05, \mathrm{n}=$ 8, HUVEC; fig. 2b).

Inhibition of SHP-2 Impairs the Ability of Endothelial Cells to Form Capillary-Like Structures in vitro and

Negatively Affects Vessel Sprouting in vivo

To investigate whether the effects seen after SHP-2 deprivation have functional consequences on vessel formation, we firstly performed Matrigel assays. Inhibition of SHP-2 with AS-ODN in HUVEC showed a $45 \pm 13 \%$ ( $3 \pm 0.8$ capillary structures $/ \mathrm{mm}^{2}, \mathrm{p}<0.01, \mathrm{n}=8$, HUVEC, fig. $3 \mathrm{~d}$, e) reduction in the number of endothelium-dependent capillary-like structures, as they detached themselves from the matrix (dark dots) and the capillary structures were incomplete compared to Rdm- 

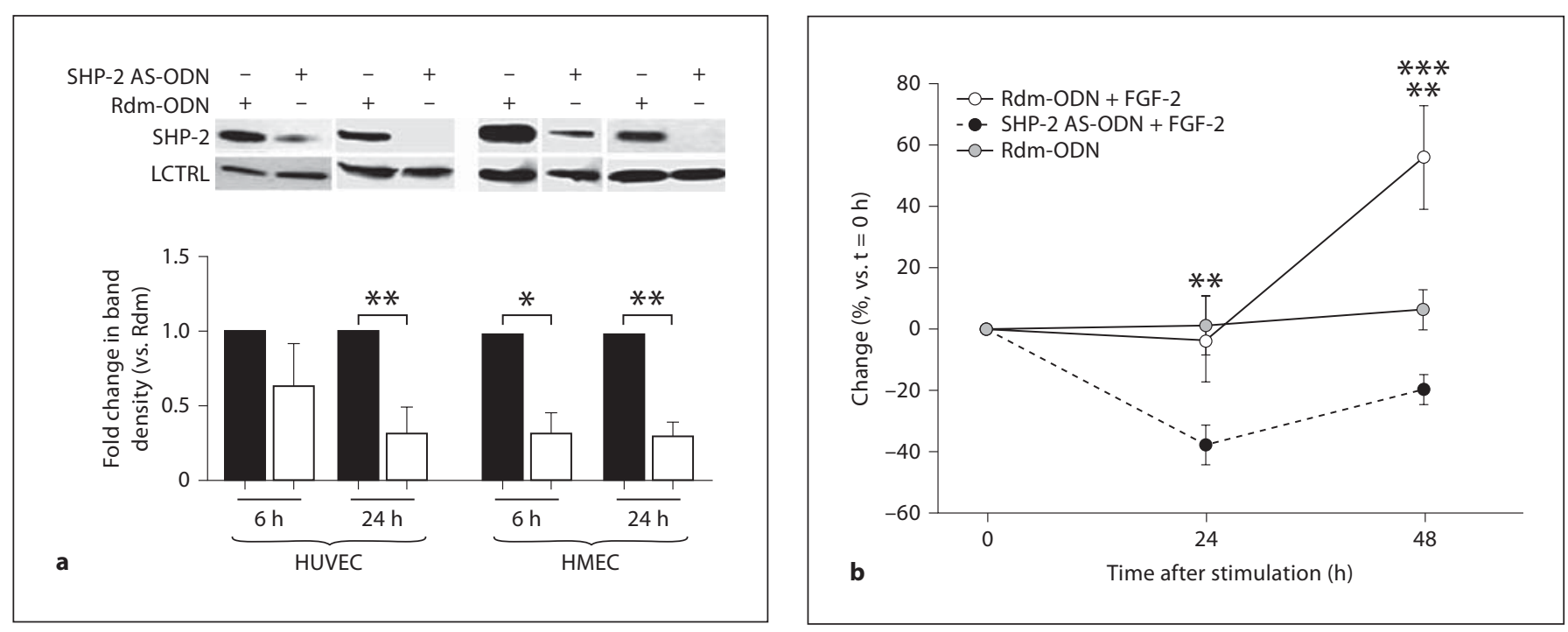

Fig. 1. Knock-down of SHP-2 protein expression and FGF-2 proliferation following SHP-2 inhibition. a SHP-2 was successfully knocked down by SHP-2 AS-ODN transfection combined with magnetofection in both HUVEC $\left({ }^{* *} \mathrm{p}<0.01, \mathrm{n}=3\right)$ and HMEC $\left({ }^{*} \mathrm{p}<0.05 ;{ }^{* *} \mathrm{p}<0.01\right.$, both $\left.\mathrm{n}=3\right)$. Immunoblots show protein expression 6 and $24 \mathrm{~h}$ following magnetofection. As a loading control (LCTRL), actin staining was performed. b FGF-2 (10 ng/ml) enhanced endothelial cell proliferation (Rdm-ODN + FGF-2), as assessed by MTT reduction of HMEC compared to non-stimulated cells (Rdm-ODN, ${ }^{* *} \mathrm{p}<0.05 ; \mathrm{n}=9,48 \mathrm{~h}$ after stimulation), whereas FGF-2 stimulation was non-effective when SHP-2 was knocked down (SHP-2 AS-ODN + FGF-2, ${ }^{* *} \mathrm{p}<$ $0.01 ; \mathrm{n}=12, \mathrm{HMEC})$. Data are normalized to the proliferative response at first measurement (before stimulation, $\mathrm{t}=0 \mathrm{~h}$ ), which is set to $0 \%$. c SHP-2 knock-down also led to a reduction in protein concentration (SHP-2 AS-ODN + FGF-2, ${ }^{* *} \mathrm{p}<0.01$; $\mathrm{n}=9$, HMEC, $48 \mathrm{~h}$ after stimulation) compared to Rdm-ODN treatment (Rdm-ODN $+\mathrm{FGF}-2, \mathrm{n}=6$ ). Data are normalized to the protein content at first measurement (before stimulation, $\mathrm{t}=0 \mathrm{~h}$ ). ${ }^{*} \mathrm{p}<0.05, \mathrm{n}=9$, Rdm-ODN vs. Rdm-ODN + FGF-2.

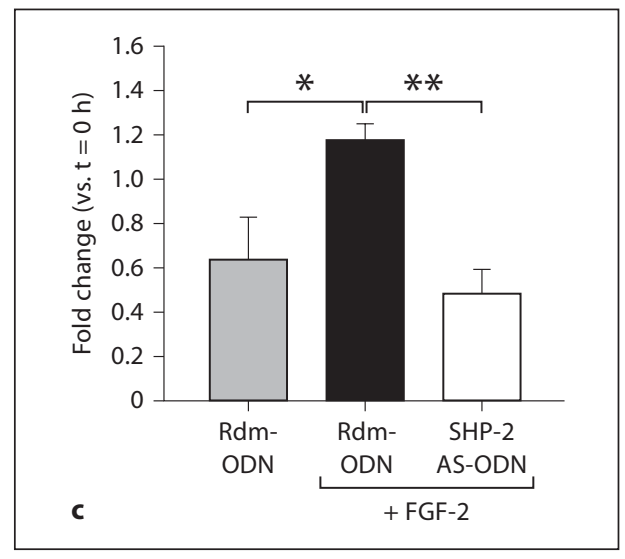

ODN-treated cells $\left(7 \pm 0.7\right.$ capillary structures $/ \mathrm{mm}^{2}$, fig. $3 \mathrm{~b}, \mathrm{e})$. In addition, treatment with the pharmacological SHP-2 inhibitor also reduced the number of capillarylike structures by $55 \pm 16 \%$ ( $4 \pm 1.6$ capillary structures/ $\mathrm{mm}^{2}, \mathrm{p}<0.05 ; \mathrm{n}=3$, HUVEC, fig. $3 \mathrm{c}$, e) compared to sham treatment (DMSO diluted $\mathrm{v} / \mathrm{v}$ to the same extent as PtpI IV; $9 \pm 0.5$ capillary structures $/ \mathrm{mm}^{2}$, fig. 3a, e).

To further investigate if SHP-2 inhibition directly influences angiogenesis, new vessel sprouting from isolated mouse aortae was assessed in Matrigel. As seen in figure $3 \mathrm{f}$ and $3 \mathrm{~g}$, treatment with PtpI IV impaired vessel formation in these experiments. The number of bifurcations of the newly formed vessels was remarkably reduced upon SHP-2 inhibition $(27 \pm 7$ bifurcations/aortic ring, $\mathrm{p}<$ $0.05 ; \mathrm{n}=10$, fig. $3 \mathrm{~g}, \mathrm{~h})$ in comparison to sham-treated aortic rings $(59 \pm 11$ bifurcations/aortic ring, $\mathrm{n}=8$, fig. 3f, h).
These findings were confirmed in vivo, using the chicken CAM assay. Treatment of the CAM with FGF-2 and VEGF-A (both $50 \mathrm{ng} / \mathrm{ml}$ ), to induce a strong angiogenic response, led to a marked increase in vessel growth (639 \pm 44 bifurcations/visual field, $\mathrm{n}=4$, fig. 3i, $\mathrm{k}$ ) compared to sham treatment $(310 \pm 29$ bifurcations/visual field; $\mathrm{p}<0.01, \mathrm{n}=5$, fig. 3i, j). Application of PtpI IV, however, drastically reduced this angiogenic effect $(334 \pm 72$ bifurcations/visual field; $\mathrm{p}<0.01 ; \mathrm{n}=4$, fig. 3i, 1 ). To rule out the possibility of the PtpI IV to be cytotoxic and therefore contribute to the observed effects, we performed a trypan blue exclusion assay with cells treated with PtpI IV and DMSO (sham). As seen in figure $3 \mathrm{~m}$, there was no increase in trypan blue-positive cells at any time compared to sham treatment $(n=7)$. This confirms that the effects seen after SHP-2 inhibition are indeed specific and not due to cytotoxicity from the solvent. 
Fig. 2. SHP-2 inhibition increases the amount of cells in SubG0 and annexin V binding. DNA content was analyzed by propidium iodide staining and apoptosis was assessed by measurement of annexin $\mathrm{V}$ binding followed by FACS analysis. a A significant increase in the SubG0 fraction $\left({ }^{* *} \mathrm{p}<0.01 ; \mathrm{n}=6\right.$, HUVEC, $24 \mathrm{~h}$ after transfection) was observed after SHP-2 AS-ODN treatment in comparison to Rdm-ODN treatment. b Annexin V antibody binding, measured by flow cytometry, was significantly increased $(* \mathrm{p}<0.05$; $\mathrm{n}=9$, HUVEC, $6 \mathrm{~h}$ after transfection) in cells where SHP-2 was knocked down (SHP-2 AS-ODN) compared to cells with normal levels of SHP-2 expression (RdmODN).

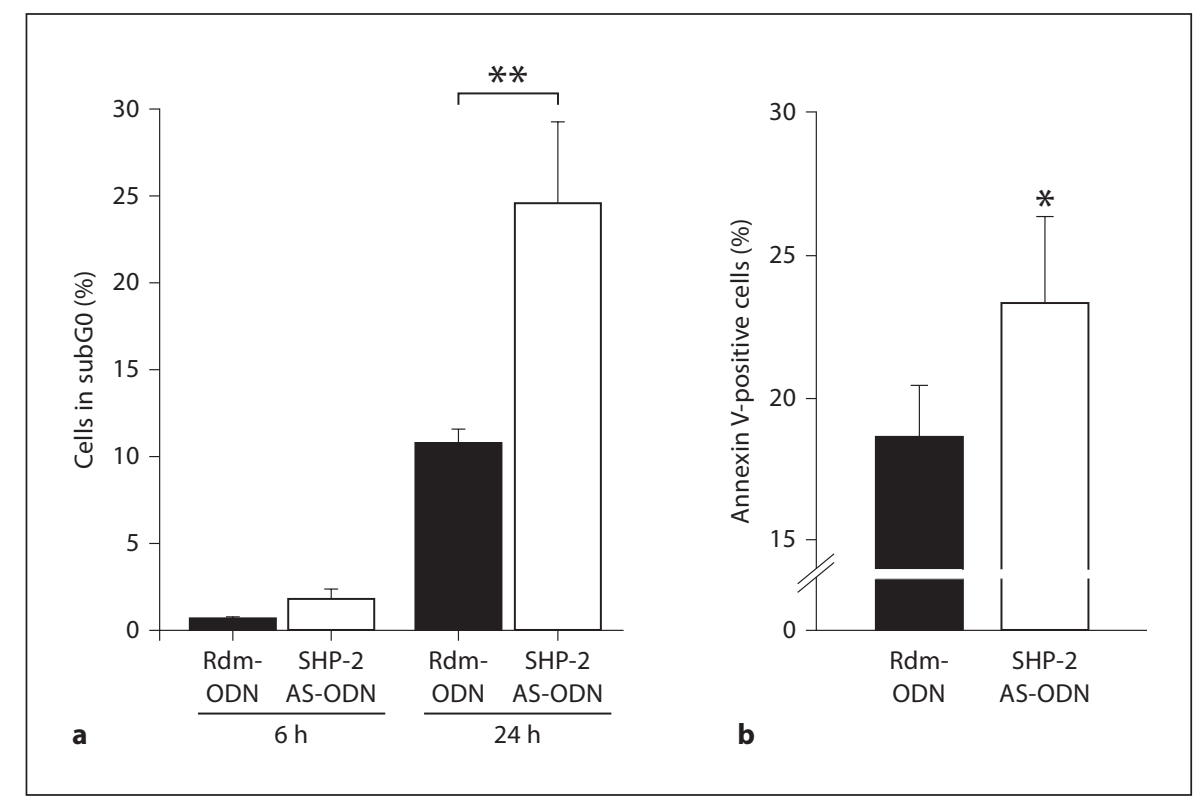

\section{SHP-2 Regulates FGF-2-Dependent Endothelial} Angiogenic Signaling Pathways

To investigate the mechanisms underlying SHP-2-dependent inhibition of endothelial angiogenesis, we tested whether SHP-2 affected activation of the PI3-K-Akt pathway, a prominent regulator of endothelial cell survival and angiogenesis $[15,29]$. Whereas tyrosine phosphorylation of the $\mathrm{p} 85$ subunit was enhanced upon stimulation with FGF-2 $(n=3)$, indicating an activation of PI3-K, it decreased upon AS-ODN treatment in comparison to Rdm-ODN (fig. 4a, $\mathrm{n}=3$, HMEC) as well as after application of PtpI IV (fig. $4 \mathrm{~b}, \mathrm{n}=4$, HMEC). Furthermore, when detecting the phosphorylation of Akt, the downstream target of PI3-K, a strong enhancement was seen after FGF-2 stimulation $(\mathrm{n}=3)$, whereas inhibition of SHP-2 led to diminished Akt phosphorylation (fig. 4c, $\mathrm{n}=4$, HMEC).

Secondly, we investigated the influence of SHP-2 on endothelial MAPK pathway activation, another eminent regulator of angiogenesis [30] and a promoter of endothelial cell survival. FGF-2 induced strong phosphorylation of ERK1/2, as revealed by immunoblotting against phospho-ERK1/2. This was decreased when inhibiting SHP-2 by SHP-2 AS-ODN ( $\mathrm{n}=5$, HMEC, fig. $5 \mathrm{a})$. To ensure the specificity of SHP-2 involvement, cells were treated with $1 \mu \mathrm{M}$ phorbol 12-myristate-13-acetate (PMA), a direct stimulator of protein kinase $\mathrm{C}$, which has been shown to activate ERK1/2 independently of SHP-2 [38]. This resulted in robust activation of ERK1/2, which, in contrast to FGF-2-dependent ERK1/2 activation, was independent of SHP-2, as SHP-2 AS-ODN had no effect in these experiments ( $\mathrm{n}=4$, HMEC, fig. $5 \mathrm{~b})$. To elucidate if PI3-K could also be an upstream regulator of the MAPK pathway and thus targeted by SHP-2 in these experiments, PI3-K was next inhibited using wortmannin (10 nM) before stimulation with FGF-2. This also led to a decrease in ERK1/2 phosphorylation regardless of FGF-2 stimulation (both $n=3$, HMEC, fig. 5c).

\section{Discussion}

Our findings highlight the potential importance of endothelial Src homology 2-containing tyrosine phosphatase SHP-2 for the formation of new blood vessels and shed light on the role of SHP-2 in angiogenic FGF-2-dependent endothelial cell signaling. Our data suggest that SHP-2 is substantial for new vessel formation in vivo, a crucial event for revascularization of ischemic tissue. We show that this involves SHP-2-dependent prevention of apoptosis of endothelial cells. Finally, our results highlight the mechanisms by which SHP-2 plays a pivotal role in angiogenic factor-dependent endothelial activation, as SHP-2 regulates the MAPK and Akt pathways upon FGF2 stimulation, which are critical for cell survival and proliferation through PI3-K.

In this study in endothelial cells, cellular proliferation observed upon FGF-2 stimulation was completely abol- 

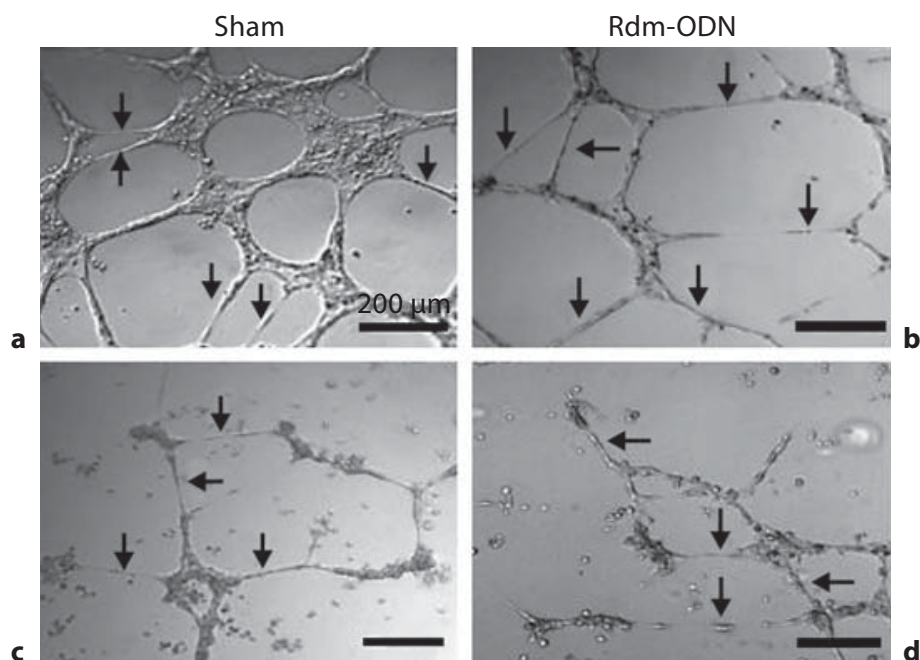

Ptpl IV

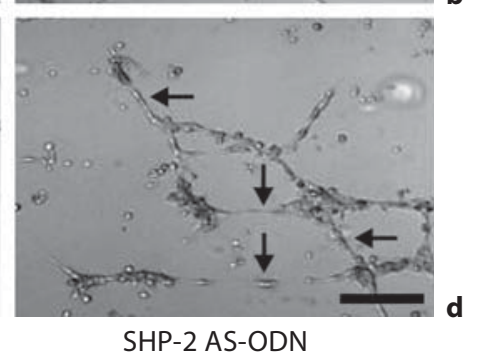

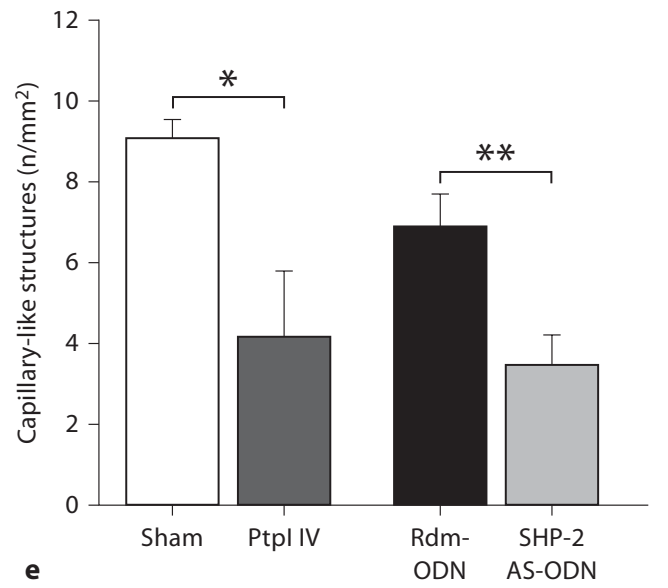
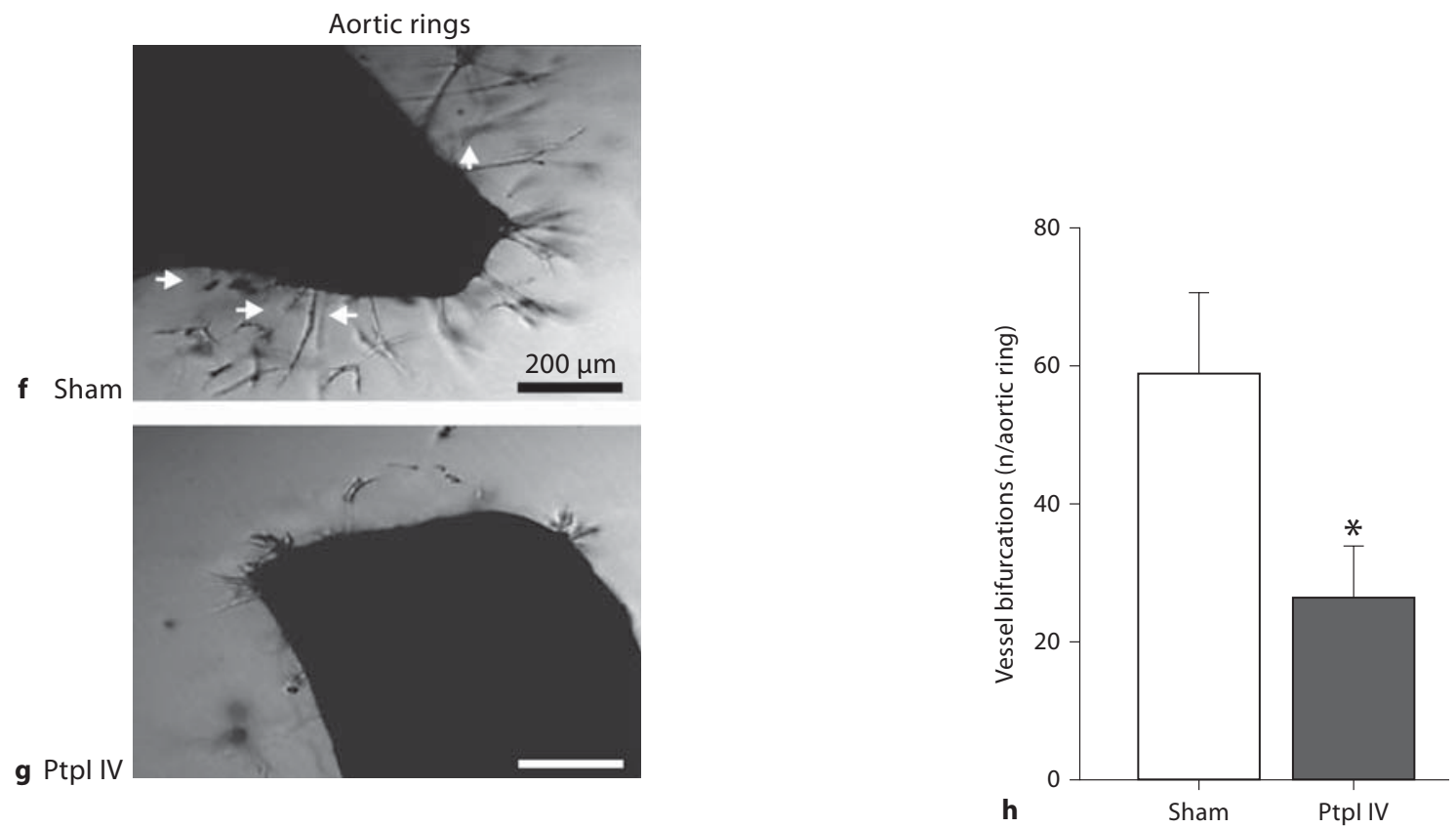

Fig. 3. Inhibition of SHP-2 impairs the ability of endothelial cells to form capillary-like structures and vessel outgrowth in vivo. a-d Representative photos of capillary-like structures (indicated by arrows, black bar in photos represents $200 \mu \mathrm{m}$ ). a HUVEC treated with sham solution. b HUVEC treated with Rdm-ODN. c HUVEC treated with PtpI IV. d HUVEC treated with SHP-2 AS-ODN. e The ability to form capillary-like sproutings in Matrigel was significantly diminished when HUVEC were treated in separate experiments with either a specific inhibitor of SHP-2 (PtpI IV, $2 \mu \mathrm{M}$ ) or SHP-2 AS-ODN compared to sham (DMSO)- treated cells $\left({ }^{*} \mathrm{p}<0.05 ; \mathrm{n}=3\right)$ or Rdm-ODN treatment, respectively $\left({ }^{* *} \mathrm{p}<0.01, \mathrm{n}=8\right)$. The structures were quantified $16 \mathrm{~h}$ following seeding of the cells. $\mathbf{f}, \mathbf{g}$ Representative photos of aortic rings $96 \mathrm{~h}$ following treatment showing the endothelial sproutings (bifurcations are indicated by white arrows, bars in photos represents $200 \mu \mathrm{m})$. f Sham (DMSO) treatment. g PtpI IV treatment. $\mathbf{h}$ The number of bifurcations on endothelial sproutings from mouse aortas was significantly lower upon SHP-2 inhibition $\left({ }^{*} \mathrm{p}<0.05, \mathrm{n}=8-10\right.$, PtpI IV). 


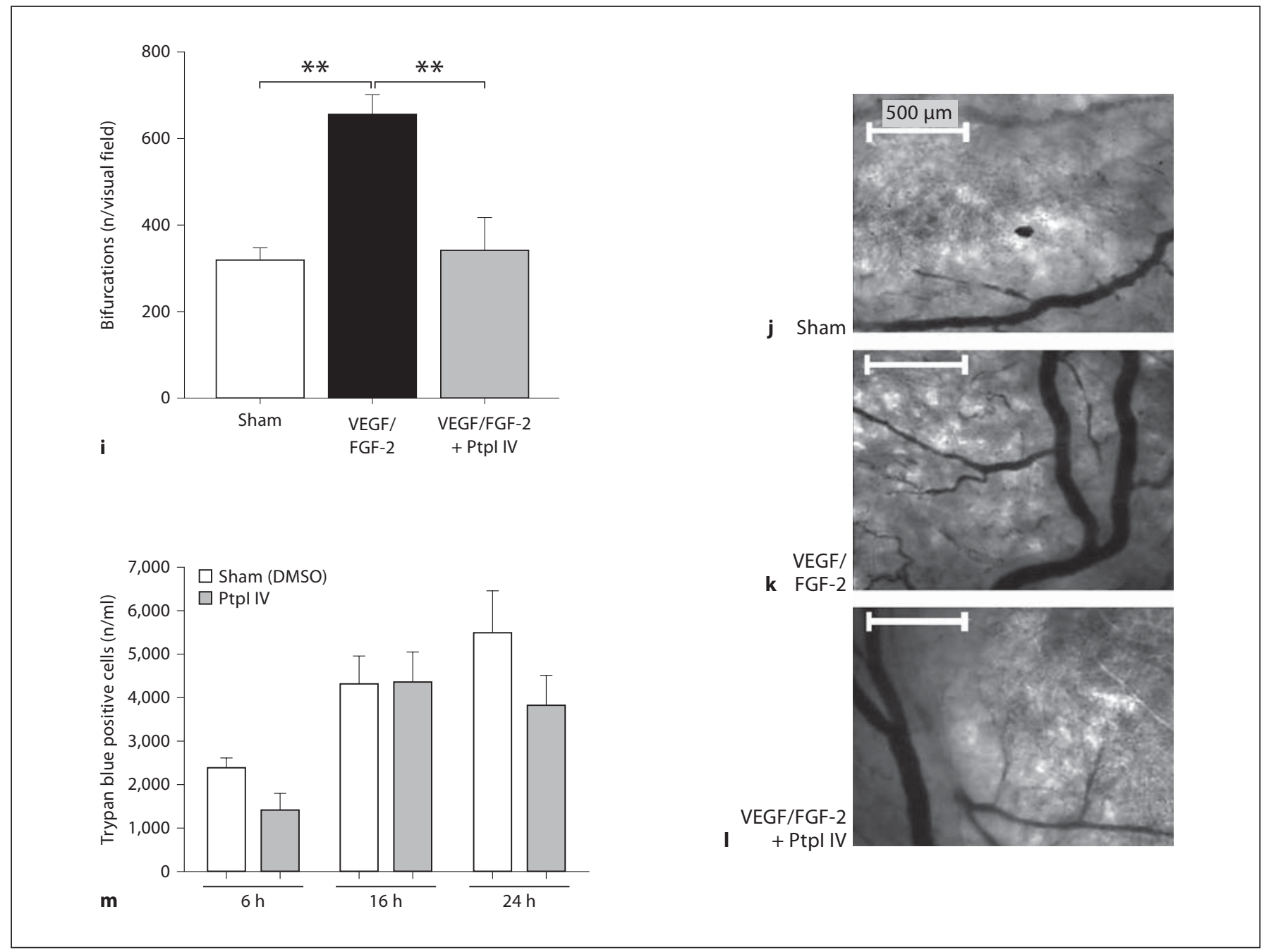

Fig. 3. $\mathbf{i}$ Vessel outgrowth in the CAM of chick embryos was severely affected by SHP-2 inhibition $\left({ }^{* *} \mathrm{p}<0.01, \mathrm{n}=4\right.$, PtpI IV). j-I Representative sections of photos of vessel outgrowth in the chicken CAM $72 \mathrm{~h}$ (white bar $=500 \mu \mathrm{m}$ ) following sham treatment (DMSO; j), VEGF + FGF-2 (both 50 ng/ml) + DMSO treat-

ished following downregulation of the tyrosine phosphatase SHP-2 using the highly specific and effective magnetofection technique [21]. As not only the cellular protein concentration but also the number of viable cells, measured by MTT reduction, were reduced after SHP-2 knockdown, we hypothesized that SHP-2 is primarily important for the preservation of endothelial cell viability. This suggestion was verified since staining the DNA of SHP-2 ASODN-treated cells with propidium iodide resulted in a peak in SubG0, indicating enhanced cell death, which was not seen in Rdm-ODN transfected cells. We confirmed this inhibitory effect on apoptosis by annexin V staining, ment $(\mathbf{k})$ and VEGF + FGF-2 + PtpI IV treatment (I). $\mathbf{m}$ The level of cytotoxicity of HMEC treated with PtpI IV $(2 \mu \mathrm{M})$ compared to sham treatment (DMSO, v/v) was assessed by trypan blue exclusion. PtpI IV treatment showed at no time an increase in nonviable cells compared to sham treatment $(n=7)$. which also revealed a significantly higher amount of proapoptotic cells following SHP-2 AS-ODN treatment, indicating that SHP-2 is necessary for endothelial cell growth and survival. This is of major importance, since endothelial cells loose their contact to the extracellular matrix, as the matrix is degraded during the initiation of the angiogenic process to allow for new sprouting, without undergoing apoptosis or anoikis. Thus, an anti-apoptotic role of SHP-2 in endothelial cells may be of significance for proper angiogenesis to occur. These data are supported by studies where SHP-2 was shown to influence growth [31] and EGF-stimulated cell cycle progression in fibroblasts [32]. 


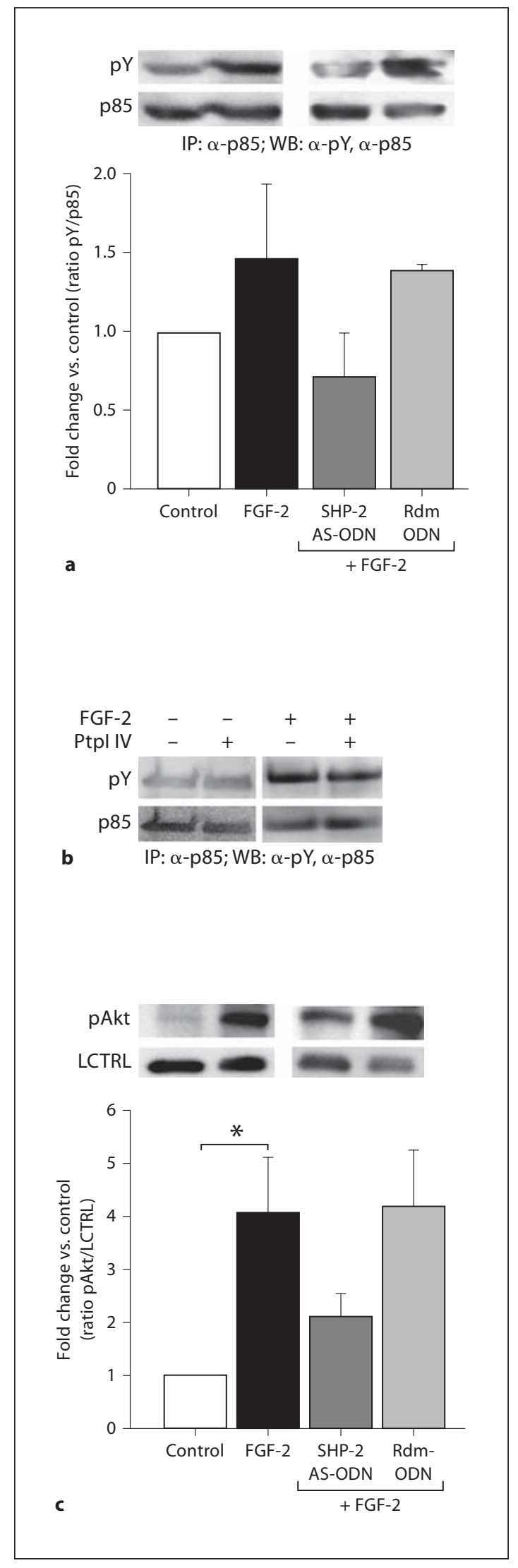

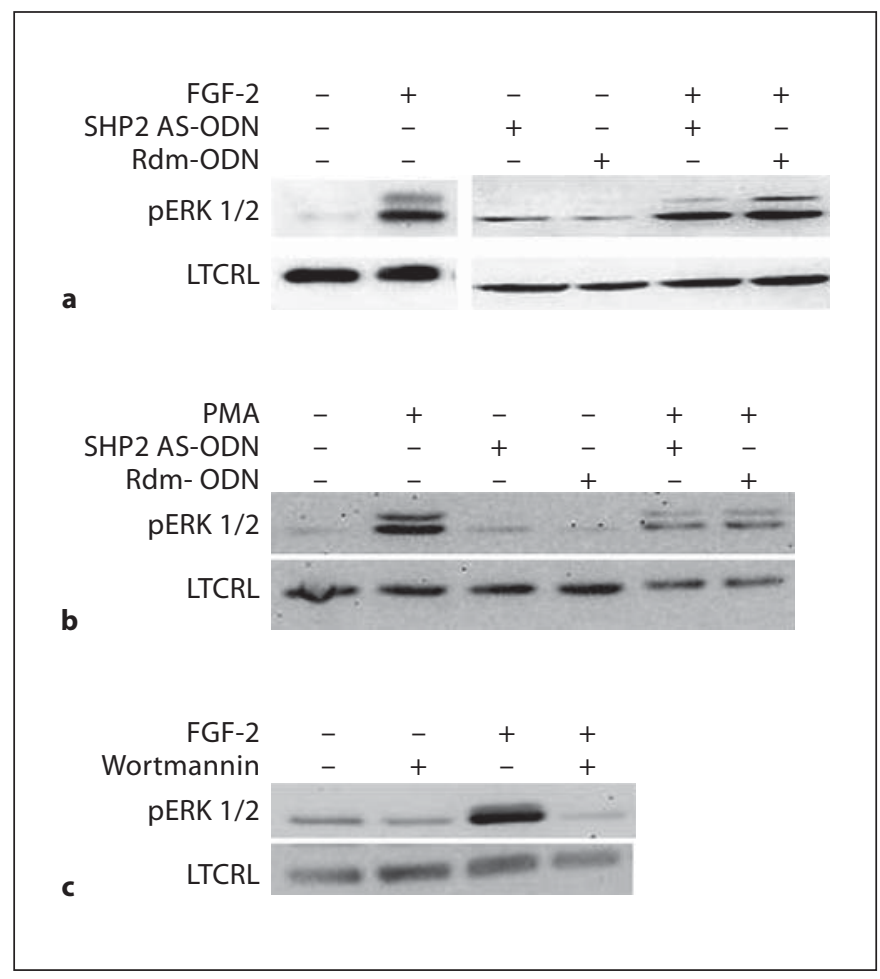

Fig. 5. SHP-2 regulates FGF-2-dependent ERK activity through PI3-K in endothelial cells. HMEC were stimulated with FGF-2 (10 $\mathrm{ng} / \mathrm{ml}$ for $10 \mathrm{~min}$ ), lysed and subjected to Western blotting. a ERK1/2 phosphorylation upon FGF-2 stimulation was prevented when SHP-2 was absent following SHP-2 AS-ODN treatment but not when Rdm-ODN was used $(\mathrm{n}=5)$. b Direct activation of ERK1/2 by protein kinase C-dependent phosphorylation (by $1 \mu$ M PMA) was not affected by SHP-2 knock-down $(\mathrm{n}=3)$. c Whereas FGF-2 induced ERK1/2 phosphorylation, wortmannin treatment followed by FGF-2 stimulation $(10 \mathrm{ng} / \mathrm{ml})$ abrogated these effects ( $\mathrm{n}=3$, HMEC). LCTRL = Loading control.

Fig. 4. SHP-2 is needed for FGF-2 activation of the endothelial PI3K-Akt pathway. HMEC were stimulated with FGF-2 (10 ng/ml for $10 \mathrm{~min}$ ), lysed and subjected to Western blotting. a Whereas FGF2 stimulation caused an increase in tyrosine phosphorylation of the p85-regulatory subunit of PI3-K, SHP-2 AS-ODN transfection led to a decrease in FGF-2-dependent phosphorylation of $\mathrm{p} 85$ $(\mathrm{n}=3)$ as assessed by immunoprecipitation of $\mathrm{p} 85$ and subsequent immunoblotting with an anti-pY antibody. b Pharmacological SHP-2 inhibition (PtpI IV, $2 \mu \mathrm{M}$ ) also reduced the FGF-2-dependent $\mathrm{p} 85$ phosphorylation $(\mathrm{n}=4)$. c Whereas FGF-2 stimulated Akt phosphorylation $\left({ }^{*} \mathrm{p}<0.05, \mathrm{n}=10\right)$, knock-down of endothelial SHP resulted in a loss of FGF-2-dependent Akt activation (anti-phospho-Akt immunoblotting, $\mathrm{n}=4$ ). Control = No stimulation; LCTRL = loading control. 
The functional anti-angiogenic consequences of SHP-2 inhibition are highlighted by the fact that knockdown as well as pharmacological inhibition of SHP-2 impaired the ability of endothelial cells to form capillary-like structures in a proangiogenic assay. Furthermore, vessel sprouting ex vivo from mouse aortae was also severely affected upon SHP-2 inhibition. Finally, inhibition of SHP-2 resulted in impaired vessel growth in the chicken CAM strongly supporting the assumption that the positive role of SHP-2 on growth or anti-apoptotic pathways is a prerequisite for angiogenesis in vivo. Previous studies showing a role for SHP-2 in cell motility by involving PECAM [9] and focal adhesion kinase [33] together with the observation that SHP-2-negative mutant endothelial cells failed to organize themselves into a highly vascularized network in the yolk sac of mouse embryos [34] support our findings that SHP-2 is an important angiogenic player.

Since the MAPK pathway is known to be an eminent regulator of apoptosis [35-37] and proliferation [37], processes highly important for angiogenesis initiation, we next investigated if SHP-2 influences ERK1/2 activation in human endothelial cells. Indeed, stimulation with the angiogenic factor FGF-2 enhanced ERK1/2 activation, whereas deprivation of SHP-2 prevented this, indicating a role for SHP-2 in FGF-2-induced MAPK pathway activation and thus prevention of endothelial apoptosis. Since treatment with PMA, a direct stimulator of protein kinase $\mathrm{C}$, for $10 \mathrm{~min}$ has been shown to activate the RafMEK (MAPK/ERK kinase)-ERK pathway independent of SHP-2 activation [38], we chose this approach to ensure that the effects of SHP-2 were specific and not an epiphenomenon of cellular apoptosis. Activation of ERK1/2 through PMA still enhanced ERK1/2 phosphorylation in spite of SHP-2 AS-ODN treatment demonstrating that the observed effects were not secondary to apoptosis. This coincides with previous findings where catalytically inactive SHP-2 inhibited FGF-induced ERK activation in Xenopus embryos [39] and where SHP-2 was required for sustained activation of MAPK by FGF in fibroblasts [34].

In addition to the MAPK pathway, our findings suggest that the PI3-K/Akt pathway, which is of major importance for endothelial survival and cell cycle progression $[40,41]$, is equally involved in the protection from apoptosis by SHP-2, because FGF-2-dependent activation of PI3-K was also diminished after SHP-2 knock-down as well as upon pharmacological inhibition. These findings are supported by data showing a role for SHP-2 in IGF-1and EGF-stimulated PI3-K activation and caspase-3-induced apoptosis in fibroblasts $[42,43]$. Moreover, SHP-2

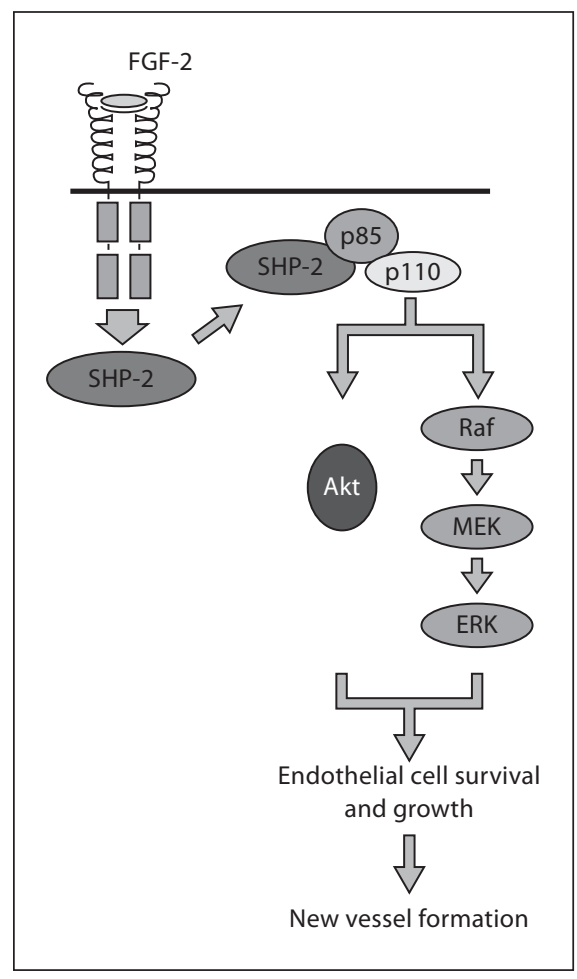

Fig. 6. FGF-2-dependent SHP-2 signaling in endothelial cells. Upon FGF-2 stimulation, the p 85 subunit of PI3-K becomes tyrosine phosphorylated, which renders the kinase active. This tyrosine phosphorylation is dependent on the presence of SHP-2. It is likely that SHP-2 and p85 are parts of the same signaling complex [43]. SHP-2-dependent PI3-K activation leads to Akt and MAPK (Raf-MEK-ERK) pathway activation. This prevents apoptosis and stimulates growth, which are prerequisites for proper vessel formation. These events were shown to be influenced by SHP-2 in this study.

AS-ODN treatment also reduced FGF-2-enhanced Akt phosphorylation in endothelial cells. Such central position of SHP-2 in the regulation of endothelial cell viability shifts the focus of the question towards the potential direct target of SHP-2 affecting these anti-apoptotic pathways. Others have reported that SHP-2 regulates ERK1/2 activity through the Src kinase by promoting the disassociation of the negative Src-regulator Csk [44, 45]. In our study, however, treatment with wortmannin together with FGF-2 resulted in decreased ERK1/2 phosphorylation. This leads to the assumption that PI3-K does not only function to activate Akt, but also activates the MAPK pathway promoting endothelial cell survival and growth. Taken together these findings demonstrate that SHP-2 regulates FGF-2-dependent ERK1/2 activation probably through PI3-K in human endothelial cells. Therefore, the regulatory subunit of PI3-K, p85, which is tyrosine phos- 
phorylated in order to activate the enzyme, thus creating a binding site for proteins containing $\mathrm{SH} 2$ domains, is one likely target or binding partner for SHP-2 in FGF-2dependent signaling. SHP-2 may in this case function as an adaptor protein recruiting PI3-K to the membrane and thus closer to its substrates. PI3-K may therefore be the link between SHP-2 and its anti-apoptotic effects observed in this study (fig. 6).

Several studies implicate the docking protein Gab-1 to be of importance for SHP-2-dependent PI3-K signaling. Indeed, SHP-2 has been found to bind Gab-1 upon FGF-2 stimulation $[46,47]$ and this seemed to positively affect Akt and ERK activation [47]. The FGF receptor substrate 2 (FRS-2) is another target for SHP-2 in FGF-dependent cell signaling [48]. SHP-2 associates with FRS-2 upon FGF stimulation resulting in tyrosine phosphorylation of SHP-2, which acts as a binding site for Grb2 [48, 49]. As recruitment of Grb2 and Sos influences Ras activation, which initiates activation of the MAPK pathway and also PI3-K [50, 51], this would be one possibility of SHP-2 to positively regulate these pathways. As SHP-2 has been shown to directly associate with the p85 subunit of PI3-K and with Gab-1 upon stimulation with several different growth factors, it is suggested that PI3-K, SHP-2 and Gab-1 constitute one signaling complex being important for PI3-K and Akt activation. In addition, Src would also constitute a suitable target for SHP-2 as it is activated by dephosphorylation of a tyrosine residue. In fact, SHP-2 has been shown to positively regulate ERK signaling through activation of Src [52].

Thus our findings identify SHP-2 as a possible central regulating enzyme in endothelial cell signaling and angiogenesis and indicate that functional SHP-2 is necessary for angiogenesis initiation by prevention of endothelial cell apoptosis. SHP-2 may therefore be of interest in the development of treatment strategies involving angiogenesis, as it may either be therapeutically prevented, when inhibition of new vessel formation is desired, or its activity or expression may be enhanced, when therapeutic angiogenesis is needed.

\section{Acknowledgments}

This work was supported by grants from the German Federal Ministry of Education and Research (Bundesministerium für Bildung und Forschung), the German Research Foundation (Deutsche Forschungsgemeinschaft) and the Friedrich Baur Foundation, Germany. The authors would like to thank Dr. F. Pogoda for valuable help with CAM assays.

\section{References}

1 Neel BG, Tonks NK: Protein tyrosine phosphatases in signal transduction. Curr Opin Cell Biol 1997;9:193-204

2 Neel BG, Gu H, Pao L: The 'Shp'ing news: $\mathrm{SH} 2$ domain-containing tyrosine phosphatases in cell signaling. Trends Biochem Sci 2003;28:284-293.

3 Qu CK: Role of the SHP-2 tyrosine phosphatase in cytokine-induced signaling and cellular response. Biochim Biophys Acta 2002; 1592:297-301.

4 Neel BG: Structure and function of SH2-domain containing tyrosine phosphatases. Semin Cell Biol 1993;4:419-432.

5 Chan RJ, Johnson SA, Li Y, et al: A definitive role of Shp-2 tyrosine phosphatase in mediating embryonic stem cell differentiation and hematopoiesis. Blood 2003;102:20742080.

6 Chen J, Yu WM, Bunting KD, et al: A negative role of SHP-2 tyrosine phosphatase in growth factor-dependent hematopoietic cell survival. Oncogene 2004;23:3659-3669.

7 Neel BG: Role of phosphatases in lymphocyte activation. Curr Opin Immunol 1997;9: 405-420.
8 Hu Y, Szente B, Kiely JM, et al: Molecular events in transmembrane signaling via E-selectin. SHP2 association, adaptor protein complex formation and ERK1/2 activation. J Biol Chem 2001;276:48549-48553.

9 O’Brien CD, Cao G, Makrigiannakis A, et al: Role of immunoreceptor tyrosine-based inhibitory motifs of PECAM-1 in PECAM-1dependent cell migration. Am J Physiol Cell Physiol 2004;287:C1103-C1113.

10 Ukropec JA, Hollinger MK, Salva SM, et al: SHP2 association with VE-cadherin complexes in human endothelial cells is regulated by thrombin. J Biol Chem 2000;275:59835986.

11 Munoz-Chapuli R, Quesada AR, Angel Medina $\mathrm{M}$ : Angiogenesis and signal transduction in endothelial cells. Cell Mol Life Sci 2004;61:2224-2243.

12 Frisch SM, Ruoslahti E: Integrins and anoikis. Curr Opin Cell Biol 1997;9:701-706.

13 Nor JE, Polverini PJ: Role of endothelial cell survival and death signals in angiogenesis. Angiogenesis 1999;3:101-116.

14 Papetti M, Herman IM: Mechanisms of normal and tumor-derived angiogenesis. Am J Physiol Cell Physiol 2002;282:C947-C970.
15 Qi JH, Matsumoto T, Huang K, et al: Phosphoinositide 3 kinase is critical for survival, mitogenesis and migration but not for differentiation of endothelial cells. Angiogenesis 1999;3:371-380.

16 Distler JH, Hirth A, Kurowska S, et al: Angiogenic and angiostatic factors in the molecular control of angiogenesis. Q J Nucl Med 2003;47:149-161.

17 Slavin J: Fibroblast growth factors: at the heart of angiogenesis. Cell Biol Int 1995;19: 431-444.

18 Tomanek RJ, Zheng W, Yue X: Growth factor activation in myocardial vascularization: therapeutic implications. Mol Cell Biochem 2004;264:3-11.

19 Krotz F, Sohn HY, Keller M, et al: Depolarization of endothelial cells enhances platelet aggregation through oxidative inactivation of endothelial NTPDase. Arterioscler Thromb Vasc Biol 2002;22:2003-2009.

20 Ades EW, Candal FJ, Swerlick RA, et al: HMEC-1: establishment of an immortalized human microvascular endothelial cell line. J Invest Dermatol 1992;99:683-690. 
21 Krotz F, de Wit C, Sohn HY, et al: Magnetofection - a highly efficient tool for antisense oligonucleotide delivery in vitro and in vivo. Mol Ther 2003;7:700-710.

22 Plank C, Schillinger U, Scherer F, et al: The magnetofection method: using magnetic force to enhance gene delivery. Biol Chem 2003;384:737-747.

23 Krotz F, Sohn HY, Gloe T, et al: Magnetofection potentiates gene delivery to cultured endothelial cells. J Vasc Res 2003;40:425-434.

24 Krotz F, Sohn HY, Gloe T, et al: NAD(P)H oxidase-dependent platelet superoxide anion release increases platelet recruitment. Blood 2002;100:917-924.

25 Mosmann T: Rapid colorimetric assay for cellular growth and survival: application to proliferation and cytotoxicity assays. J Immunol Methods 1983;65:55-63.

26 Crompton T, Peitsch MC, MacDonald HR, et al: Propidium iodide staining correlates with the extent of DNA degradation in isolated nuclei. Biochem Biophys Res Commun 1992; 183:532-537.

27 Huang P, Ramphal J, Wei J, et al: Structurebased design and discovery of novel inhibitors of protein tyrosine phosphatases. Bioorg Med Chem 2003;11:1835-1849.

28 Brooks PC, Montgomery AM, Cheresh DA: Use of the 10-day-old chick embryo model for studying angiogenesis. Methods Mol Biol 1999;129:257-269.

29 Nakashio A, Fujita N, Tsuruo T: Topotecan inhibits VEGF- and bFGF-induced vascular endothelial cell migration via downregulation of the PI3K-Akt signaling pathway. Int J Cancer 2002;98:36-41.

30 Hood JD, Bednarski M, Frausto R, et al: Tumor regression by targeted gene delivery to the neovasculature. Science 2002;296:24042407.

31 Shi ZQ, Yu DH, Park M, et al: Molecular mechanism for the Shp-2 tyrosine phosphatase function in promoting growth factor stimulation of Erk activity. Mol Cell Biol 2000;20:1526-1536.
32 Bennett AM, Hausdorff SF, O'Reilly AM, et al: Multiple requirements for SHPTP2 in epidermal growth factor-mediated cell cycle progression. Mol Cell Biol 1996;16:11891202.

33 Qi JH, Ito N, Claesson-Welsh L: Tyrosine phosphatase SHP-2 is involved in regulation of platelet-derived growth factor-induced migration. J Biol Chem 1999;274:1445514463.

34 Saxton TM, Henkemeyer M, Gasca S, et al: Abnormal mesoderm patterning in mouse embryos mutant for the $\mathrm{SH} 2$ tyrosine phosphatase Shp-2. EMBO J 1997;16:2352-2364.

35 Slater EP, Stubig T, Lau QC, et al: C-Raf controlled pathways in the protection of tumor cells from apoptosis. Int J Cancer 2003;104: 425-432.

36 Alavi A, Hood JD, Frausto R, et al: Role of Raf in vascular protection from distinct apoptotic stimuli. Science 2003;301:94-96.

37 Chang F, Steelman LS, Shelton JG, et al: Regulation of cell cycle progression and apoptosis by the Ras/Raf/MEK/ERK pathway (review). Int J Oncol 2003;22:469-480.

38 Noguchi T, Matozaki T, Horita K, et al: Role of SH-PTP2, a protein-tyrosine phosphatase with Src homology 2 domains, in insulinstimulated Ras activation. Mol Cell Biol 1994;14:6674-6682.

39 Tang TL, Freeman RM, O'Reilly AM, et al: The SH2-containing protein-tyrosine phosphatase SH-PTP2 is required upstream of MAP kinase for early Xenopus development. Cell 1995;80:473-483.

40 Shiojima I, Walsh K: Role of Akt signaling in vascular homeostasis and angiogenesis. Circ Res 2002;90:1243-1250.

41 Thakker GD, Hajjar DP, Muller WA, et al: The role of phosphatidylinositol 3-kinase in vascular endothelial growth factor signaling. J Biol Chem 1999;274:10002-10007.

42 Ivins Zito C, Kontaridis MI, Fornaro M, et al: SHP-2 regulates the phosphatidylinositide 3'-kinase/Akt pathway and suppresses caspase 3-mediated apoptosis. J Cell Physiol 2004;199:227-236.
43 Wu CJ, O'Rourke DM, Feng GS, et al: The tyrosine phosphatase SHP-2 is required for mediating phosphatidylinositol 3-kinase/ Akt activation by growth factors. Oncogene 2001;20:6018-6025.

44 Ren Y, Meng S, Mei L, et al: Roles of Gabl and SHP2 in paxillin tyrosine dephosphorylation and Src activation in response to epidermal growth factor. J Biol Chem 2004;279: 8497-8505.

45 Zhang SQ, Yang W, Kontaridis MI, et al: Shp2 regulates SRC family kinase activity and Ras/Erk activation by controlling Csk recruitment. Mol Cell 2004;13:341-355.

46 Ong SH, Hadari YR, Gotoh N, et al: Stimulation of phosphatidylinositol 3-kinase by fibroblast growth factor receptors is mediated by coordinated recruitment of multiple docking proteins. Proc Natl Acad Sci USA 2001;98:6074-6079.

47 Zhang SQ, Tsiaras WG, Araki T, et al: Receptor-specific regulation of phosphatidylinositol 3'-kinase activation by the protein tyrosine phosphatase Shp2. Mol Cell Biol 2002; 22:4062-4072.

48 Hadari YR, Kouhara H, Lax I, et al: Binding of Shp2 tyrosine phosphatase to FRS2 is essential for fibroblast growth factor-induced PC12 cell differentiation. Mol Cell Biol 1998; 18:3966-3973.

49 Kontaridis MI, Liu X, Zhang L, et al: Role of SHP-2 in fibroblast growth factor receptormediated suppression of myogenesis in C2C12 myoblasts. Mol Cell Biol 2002;22: 3875-3891.

50 Chang F, Steelman LS, Lee JT, et al: Signal transduction mediated by the Ras/Raf/ MEK/ERK pathway from cytokine receptors to transcription factors: potential targeting for therapeutic intervention. Leukemia 2003; 17:1263-1293.

51 Yart A, Mayeux P, Raynal P: Gab1, SHP-2 and other novel regulators of Ras: targets for anticancer drug discovery? Curr Cancer Drug Targets 2003;3:177-192.

52 Cunnick JM, Meng S, Ren Y, et al: Regulation of the mitogen-activated protein kinase signaling pathway by SHP2. J Biol Chem 2002; 277:9498-9504. 